\title{
Downregulation of microRNA-139 is associated with hepatocellular carcinoma risk and short-term survival
}

\author{
TAO LI* ${ }^{*}$, JIKAI YIN* ${ }^{*}$ LIJUAN YUAN, SHOULI WANG, LIN YANG, XILIN DU and JIANGUO LU \\ Department of General Surgery, Tangdu Hospital of The Fourth Military Medical University, \\ Xi'an, Shaanxi 710038, P.R. China
}

Received January 14, 2014; Accepted February 7, 2014

DOI: $10.3892 /$ or.2014.3032

\begin{abstract}
MicroRNAs (miRNAs) have been frequently reported to be diagnostic biomarkers and prognostic factors for cancer. The aim of the present study was to evaluate the clinical significance of microRNA-139 (miRNA-139) in hepatocellular carcinoma (HCC). All 31 patients enrolled in the present study had received curative hepatectomy. The objective miRNA was determined using miRNA microarray. The miRNA-139 expression level in cancerous tissue specimens was measured by means of reverse transcription and quantitative polymerase chain reaction, and compared with that in 31 corresponding peritumoral non-cancerous tissues. Plasma miRNA-139 expression was also quantified. The diagnostic value of plasma miRNA-139 for differentiating patients with HCC from the ones with chronic $\mathrm{HBV}$-hepatitis $(\mathrm{CH})$ was analyzed. The miRNA microarray performed in 3 pairs of tissue specimens determined miRNA-139 was downregulated $(\mathrm{p}=0.017)$. Compared with plasma of chronic HBV-hepatitis, miRNA-139 was lowly expressed in plasma of HCC patients $(\mathrm{p}<0.010)$. ROC analysis of plasma miRNA-139 yielded an AUC of $0.764(\mathrm{p}<0.010)$ with sensitivity of $80.6 \%$ and specificity of $58.1 \%$ while differentiating HCC from chronic HBV-hepatitis. The diagnostic power of serum $\alpha$-fetoprotein (AFP) was also evaluated. The combination of miRNA-139 and AFP improved the differentiating power. Subsequently, 31 HCC patients were divided into the low or high expression group based on plasma miRNA-139 level. Plasma miRNA-139 expression was correlated with serum AFP ( $\mathrm{p}=0.043)$, Edmondson-Steiner grading $(\mathrm{p}=0.038)$. In addition, there was a significant difference in the 1-year survival rates between the two groups $(\mathrm{p}=0.023)$. miRNA-139 was downregulated in the cancerous tissue and
\end{abstract}

Correspondence to: Professor Jianguo Lu or Professor Xilin Du, Department of General Surgery, Tangdu Hospital of The Fourth Military Medical University, 1 Xin'si Road, Ba'qiao, Xi'an, Shaanxi 710038, P.R. China

E-mail: lujguo@hotmail.com

E-mail: dxlin0705@163.com

*Contributed equally

Key words: hepatocellular carcinoma, microRNA, microRNA-139, biomarker, survival analysis plasma of HCC patients. Plasma miRNA-139 is a diagnostic biomarker and prognostic factor for HCC.

\section{Introduction}

Hepatocellular carcinoma (HCC), the fifth in incidence among malignant tumors, accounts for 70-85\% of primary liver cancer cases. Each year, 748,000 cases are newly diagnosed as HCC worldwide, and over half of them are in China (1), where HCC is the second most frequent cause for cancer-related mortality since the 1990s (2). HBV infection is the primary etiology that leads to liver cirrhosis-related carcinoma (3). The reason for the high mortality in HCC is that the tumor is always detected at advanced stages when curative therapy cannot be carried out due to intrahepatic or extrahepatic metastases. Although serum $\alpha$-fetoprotein (AFP) has been widely accepted and used as a serous biomarker for screening HCC in a high risk population for years, the sensitivity and specificity of serum AFP only ranged from 40-65 and 76-96\%, respectively (4), suggesting it may not be an ideal indicator to identify HCC from other diseases. Therefore, discovering new biomarkers for early diagnosis of $\mathrm{HCC}$ are still needed in clinical practice.

Mature microRNAs (miRNAs) are 19- to 25-nt transcripts of small non-coding RNA family processed from 70- to 100-nt hairpin-shaped precursors. The sequences of some miRNAs are conserved in various biological species, suggesting that these tiny molecules participate in essential processes of development, proliferation, differentiation and/or apoptosis in organisms (5). Depending on certain cellular functions of their targets, dysregulation of miRNAs may play oncogenic roles, such as Myc, or tumor suppressor roles, such as p53, to induce or inhibit tumorigenesis (6). Meanwhile, the importance of miRNAs in cancer progression has been reported, as miRNAs can both influence the effect of chemotherapy (7) as well as the development of drug resistance (8). Although the precise biological functions of miRNAs are not yet fully understood, some studies demonstrated that miRNA expression profile is distinguished in diverse diseases, indicating that miRNAs may be used as biomarkers for cancer diagnosis and prognosis prediction $(9,10)$. Whether those dysregulated miRNAs are related to $\mathrm{HCC}$ and/or as a general mechanism in disease progression to cancer is an important concern for utilization of miRNAs as biomarkers for HCC risk, treatment response and clinical outcome prediction (11). 
In the present study, we investigated comprehensive miRNA expression profiling of HCC using miRNA microarray in hepatocellular cancerous tissue and peritumoral noncancerous tissue, and found that microRNA-139 (miRNA-139) was significantly downregulated in hepatocellular cancerous tissue. Furthermore, we detected and verified the miRNA-139 expression level in tissue and blood to investigate the correlation between miRNA-139 and clinical characteristics to identify the diagnostic and prognostic values of miRNA-139 in $\mathrm{HCC}$ patients.

\section{Materials and methods}

Patients, samples and data collection. The study population was enrolled at the general surgery department of Tangdu Hospital affiliated to the Fourth Military Medical University (Xi'an, China). Among the study population, 31 patients were newly diagnosed and histologically confirmed HCC, and subsequently received curative hepatectomy according to the National Comprehensive Cancer Network (NCCN) guidelines for hepatobiliary cancer. Cancerous tissue samples (CT group) were collected from surgery and pathologically confirmed as $\mathrm{HCC}$; peritumoral non-cancerous tissue samples (NT group) were collected from normal liver tissue at $3 \mathrm{~cm}$ beyond the tumor margin. Plasma samples (PS group) were obtained from patients prior to surgery, while the matched plasma samples (MPS group) were obtained from 31 age- and gender-matched chronic HBV-hepatitis $(\mathrm{CH})$ patients. The clinical characteristics of all subjects are summarized in Table I. All study subjects provided informed consent, and the present study was approved by the Review Board of Tangdu Hospital Ethics Committee.

miRNA microarray. miRNA microarray was performed in three pairs of tissue specimens which were collected from two male and one female HCC patients, using a service provider (LC Sciences, USA). The assay started from 4 to $8 \mu \mathrm{g}$ total RNA sample and was 3 '-extended with a poly(A) tail using poly(A) polymerase. An oligonucleotide tag was then liquated to the poly(A) tail for later fluorescent dye staining. Hybridization was performed overnight on a $\mu$ Paraflo microfluidic chip using a micro-circulation pump (Atactic Technologies, USA). On the microfluidic chip, each detection probe consisted of a chemically modified nucleotide coding segment complementary to target miRNA or control RNA and a spacer segment of polyethylene glycol to extend the coding segment away from the substrate. The detection probes were made by in situ synthesis using photogenerated reagent chemistry. Hybridization used $10016 \mathrm{X} \mathrm{SSPE}$ buffer containing $25 \%$ formamide at $34^{\circ} \mathrm{C}$. After RNA hybridization, tag-conjugating $\mathrm{Cy} 3$ dye was circulated through the microfluidic chip for dye staining. Fluorescence images were collected using a laser scanner (GenePix 4000B; Molecular Devices, USA) and digitized using Array-Pro image analysis software (Media Cybernetics, USA). Data were analyzed by first subtracting the background and then normalizing the signals using a LOWESS filter (locally-weighted regression).

Total RNA extraction and quantification. All tissue samples were quick-frozen in liquid nitrogen and stored at $-80^{\circ} \mathrm{C}$ immediately after removal. The isolation of tissue total RNA was completed using miRNeasy Mini kit (Qiagen, Germany). While extracting RNA, $25 \mathrm{mg}$ frozen tissue with liquid nitrogen was ground to fine powder manually by mortar and pestle. After transferring the fine powder into an Eppendorf tube instantly, $700 \mu \mathrm{l}$ QIAzol lysis reagent and $140 \mu \mathrm{l}$ chloroform was added into the tube. The mixture was centrifuged at $12,000 \mathrm{rpm}$ (revolutions/min) for $15 \mathrm{~min}$ at $4^{\circ} \mathrm{C}$, and the aqueous phase, together with 1.5 volumes of $100 \%$ ethanol, was pipeted into a fresh tube. Then, the sample was purified in RNeasy Mini column using $700 \mu \mathrm{l}$ buffer RWT once and $500 \mu \mathrm{l}$ buffer RPE twice. Finally, $50 \mu \mathrm{l}$ RNase-free $\mathrm{dd}_{2} \mathrm{O}$ was used to elute total RNA from the RNeasy Mini column.

The plasma samples were isolated from whole blood before storing. In order to completely remove cell debris, the fresh whole blood was centrifuged at 1,600 rpm for $5 \mathrm{~min}$, followed by $13,000 \mathrm{rpm}$ for another $15 \mathrm{~min}$. The plasma was stored in a cryogenic tube at $-80^{\circ} \mathrm{C}$. The miRNeasy Serum/Plasma kit provided by Qiagen was used to carry out extraction of total RNA from plasma according to the manufacturer's instructions. The purity and concentration of total RNA preparations were determined by measuring the absorbance at UV $260 \mathrm{~nm}$ (A260) and UV $280 \mathrm{~nm}$ (A280) in a spectrophotometer (BioTek Epoch, USA). Pure RNA solution has an A260/A280 ratio of 1.9-2.1. All RNA preparations were stored at $-80^{\circ} \mathrm{C}$.

Reverse transcription and quantitative polymerase chain reaction ( $R T-q P C R)$. Quantification of mature miRNAs was accomplished by a two-step method. Firstly, RNA was 3'extended with a poly(A) tail using poly(A) polymerase, then the poly(A) product was reverse transcribed using oligo(dT)Universal Tag (Tiangen, China). Subsequently, real-time quantitative PCR was performed with miRNA-139 primer and internal normalization primer according to the procedures of miRcute miRNA SYBR-Green qPCR detection kit (Tiangen) in Mx3000p sequence detection system (Agilent, USA), using the following conditions: $94^{\circ} \mathrm{C}$ for a 2 -min cycle, followed by 45 cycles of $94^{\circ} \mathrm{C}$ for $20 \mathrm{sec}$, and $60^{\circ} \mathrm{C}$ for $34 \mathrm{sec}$. Briefly, $20 \mu \mathrm{l}$ PCR system contained $2 \mu \mathrm{l}$ of RT product solution, $10 \mu \mathrm{l}$ of 2X miRcute miRNA Premix (including SYBR), $0.4 \mu \mathrm{l}$ forward primer, $0.4 \mu \mathrm{l}$ reverse primer and $7.2 \mu \mathrm{l}$ RNase-free $\mathrm{ddH}_{2} \mathrm{O}$. Triplicate PCRs were carried out for every cDNA sample, including negative controls without templates. hsa-miR-U6 and hsa-miR-16 were used as the internal normalization control for tissue and plasma sample respectively. All primers were designed and provided by Tiangen. The expression level of miRNA was computed using the comparative $\Delta \mathrm{Ct}$ method as previously reported (12).

Statistical analysis. Data analysis was performed by software SPSS 21.0 for Windows (IBM SPSS, USA). The difference of miRNA expression levels between groups was calculated using the t-test or Mann-Whitney U test. The Pearson's correlation coefficient was used to calculate correlations. The diagnostic value for differentiating HCC patients from chronic HBV-hepatitis patients was evaluated by receiver operator characteristic curve (ROC curve) and the areas under ROC curve (AUC). The clinicopathological data were represented as means \pm SD or frequencies, and differences between groups were calculated by the t-test, Mann-Whitney U test or Fisher's exact test. The Kaplan-Meier method was applied to determine 
Table I. Clinicopathological characteristics of hepatocellular carcinoma (HCC) patients and chronic HBV-hepatitis (CH) patients.

\begin{tabular}{|c|c|c|c|}
\hline & \multicolumn{2}{|c|}{ Patients with different diseases } & \multirow[b]{2}{*}{ P-value } \\
\hline & $\mathrm{HCC}(\mathrm{n}=31)$ & $\mathrm{CH}(\mathrm{n}=31)$ & \\
\hline \multicolumn{4}{|l|}{ Clinical factors } \\
\hline Age (years) & $49 \pm 11$ & $49 \pm 10$ & 0.981 \\
\hline Gender (male/female) & $26 / 5$ & $26 / 5$ & 0.999 \\
\hline WBC $\left(\times 10^{9} / 1\right)$ & $6.06 \pm 2.48$ & $5.51 \pm 2.21$ & 0.358 \\
\hline $\operatorname{RBC}\left(\mathrm{x} 10^{12} / \mathrm{l}\right)$ & $4.54 \pm 0.78$ & $4.49 \pm 0.81$ & 0.830 \\
\hline $\operatorname{PLT}\left(\times 10^{9} / 1\right)$ & $128.90 \pm 59.10$ & $131.84 \pm 60.26$ & 0.847 \\
\hline $\mathrm{Hb}(\mathrm{g} / \mathrm{l})$ & $139.87 \pm 20.48$ & $140.74 \pm 21.30$ & 0.870 \\
\hline $\operatorname{ALB}(g / l)$ & $40.25 \pm 4.32$ & $40.96 \pm 8.78$ & 0.684 \\
\hline $\mathrm{TB}(\mu \mathrm{mol} / \mathrm{l})$ & $20.17 \pm 7.88$ & $22.17 \pm 12.66$ & 0.458 \\
\hline $\mathrm{DB}(\mu \mathrm{mol} / \mathrm{l})$ & $5.71 \pm 4.15$ & $7.05 \pm 4.35$ & 0.220 \\
\hline $\mathrm{IB}(\mu \mathrm{mol} / \mathrm{l})$ & $14.14 \pm 5.75$ & $15.13 \pm 9.40$ & 0.620 \\
\hline $\operatorname{ALT}(\mathrm{U} / \mathrm{l})$ & $45.42 \pm 22.53$ & $45.39 \pm 29.88$ & 0.996 \\
\hline AST (U/l) & $57.23 \pm 30.47$ & $43.00 \pm 33.00$ & 0.115 \\
\hline $\operatorname{ALP}(\mathrm{U} / \mathrm{l})$ & $93.00 \pm 61.00$ & $81.50 \pm 61.75$ & 0.134 \\
\hline GGT (U/l) & $67.00 \pm 55.00$ & $59.00 \pm 73.25$ & 0.714 \\
\hline Operation time (min) & $194 \pm 42$ & & \\
\hline Blood loss (ml) & $740.65 \pm 582.33$ & & \\
\hline Liver cirrhosis $(+/-)$ & $20 / 11$ & & \\
\hline Hypersplenism (+/-) & $16 / 15$ & & \\
\hline \multicolumn{4}{|l|}{ Tumor-related factors } \\
\hline AFP (ng/ml) & $17.80 \pm 598.60$ & $7.10 \pm 13.10$ & $0.007^{\mathrm{a}}$ \\
\hline $\mathrm{CEA}(\mathrm{ng} / \mathrm{ml})$ & $2.57 \pm 1.81$ & $2.15 \pm 1.32$ & 0.311 \\
\hline CA19-9 (U/ml) & $20.97 \pm 15.86$ & $16.50 \pm 33.00$ & 0.749 \\
\hline Child-Pugh grading (A/B) & $26 / 5$ & $19 / 12$ & 0.086 \\
\hline Tumor no. (single/multiple) & $26 / 5$ & & \\
\hline Tumor size $(\mathrm{cm})$ & $8.24 \pm 3.20$ & & \\
\hline Vascular invasion $(+/-)$ & $2 / 29$ & & \\
\hline Edmondson-Steiner grading $(\mathrm{I} / \mathrm{II}+\mathrm{III})$ & $5 / 26$ & & \\
\hline TNM staging (I/II/III) & $12 / 3 / 16$ & & \\
\hline Okuda staging $(1 / 2)$ & $14 / 17$ & & \\
\hline BCLC staging $(\mathrm{A} / \mathrm{B} / \mathrm{C})$ & $14 / 8 / 9$ & & \\
\hline CLIP scoring (1/2/3) & $15 / 13 / 3$ & & \\
\hline
\end{tabular}

There is no significant difference in terms of demographic characteristics (age and gender), ALT ( $\mathrm{p}=0.996)$, AST $(\mathrm{p}=0.115)$, TB $(\mathrm{p}=0.458), \mathrm{CEA}$ $(\mathrm{p}=0.311), \mathrm{CA} 19-9(\mathrm{p}=0.749)$ and Child-Pugh grading $(\mathrm{p}=0.086)$. Despite the significant difference in serum AFP value $(\mathrm{p}=0.007)$ between the two groups, the two groups are fully comparable in the present study. ${ }^{\mathrm{a}} \mathrm{p}<0.010$. WBC, white blood cell; RBC, red blood count; PLT, platelets; $\mathrm{Hb}$, hemoglobin; ALB, albumin; TB, total bilirubin; DB, direct bilirubin; IB, indirect bilirubin; ALT, alanine transaminase; AST, aspartate aminotransferase; ALP, alkaline phosphatase; GGT, $\gamma$-glutamyl transpeptidase; AFP, $\alpha$-fetoprotein; CEA, carcinoembryonic antigen; CA19-9, carbohydrate antigen 19-9; BCLC staging, Barcelona Clinic Liver Cancer staging; CLIP scoring, the Cancer of the Liver Italian Program scoring.

1-year survival rate, and the statistical difference between two groups was analyzed using Cox's proportional hazard model. A value of $\mathrm{p}<0.050$ was considered to indicate statistically significant differences.

\section{Results}

Patient characteristics. There were $31 \mathrm{HCC}$ patients $(26$ males and 5 females) with a mean age of 49 years (SD, 11; range,
27-69 years) while another 31 age- and gender-matched $\mathrm{CH}$ patients with a mean age of 49 years (SD, 10; range, 27-69 years) were enrolled in the present study. There was no significant difference in terms of demographic characteristics (age and gender), ALT ( $\mathrm{p}=0.996)$ AST $(\mathrm{p}=0.115)$, TB $(\mathrm{p}=0.458)$, CEA ( $p=0.311)$, CA19-9 ( $\mathrm{p}=0.749)$, and Child-Pugh grading $(\mathrm{p}=0.086)$. There was a significant difference in serum AFP value $(p=0.007)$ between the two groups (Table I). Despite the difference in AFP value, the two groups were completely 


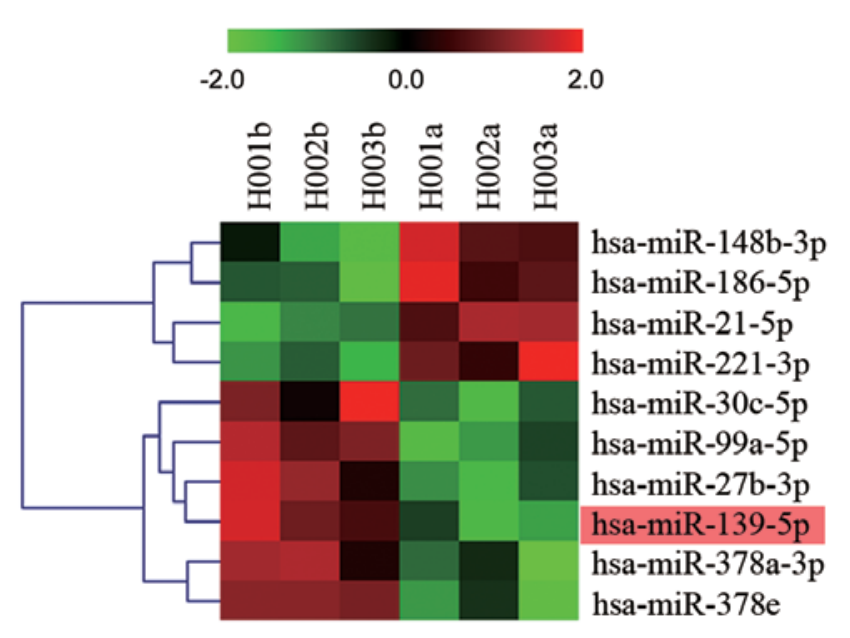

Figure 1. Heat map showing the comprehensive miRNA expression profiling of HCC.

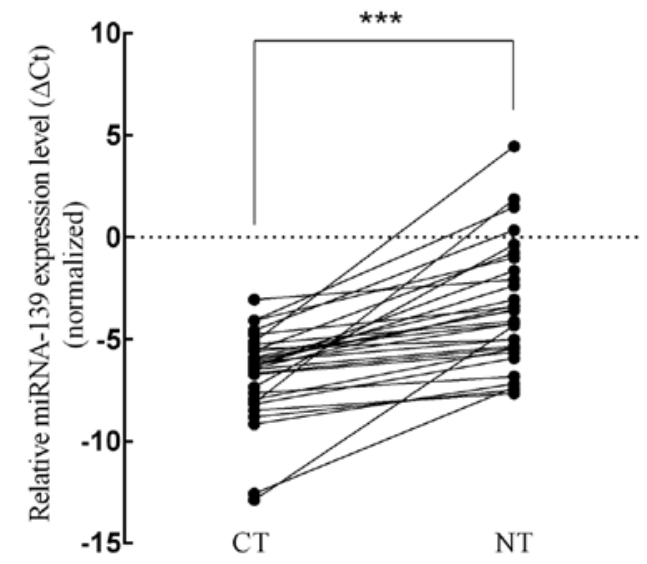

Figure 2. The average $\Delta \mathrm{Ct}$ value of miRNA-139 in cancerous tissue was -6.704 , which was significantly lower than that of -3.490 in non-cancerous tissue. ${ }^{* * *} \mathrm{p}<0.001$.

comparable in the present study. All HCC patients received curative hepatectomy during 2010-2012 with a median survival time of 362 days ( \pm 160 days).

\section{Association between miRNA-139 and HCC risk}

Ten miRNAs dysregulated in HCC. We hypothesized that miRNAs may participate in liver tumorigenesis, and are aberrantly expressed in HCC samples. The miRNA microarray was performed in 3 hepatocellular cancerous tissues and corresponding paired non-cancerous tissues. Fig. 1 shows the heat map of these dysregulated targets and defined comprehensive miRNA expression profiling. The most conspicuous underexpression values were found for miRNA-139, miRNA-99a, miRNA-27b, miRNA-378a, miRNA-378e and miRNA-30c, while the overexpression values were found for miRNA-21, miRNA-221, miRNA-148b and miRNA-186.

miRNA-139 is downregulated in HCC tissue and plasma samples. Among these dysregulated miRNAs, miRNA-139 expression level was further analyzed in the CT and NT group by RT-qPCR to confirm the dysregulation in tumoral tissues. We found that miRNA-139 expression level was significantly lower in the patients of the CT group, compared to

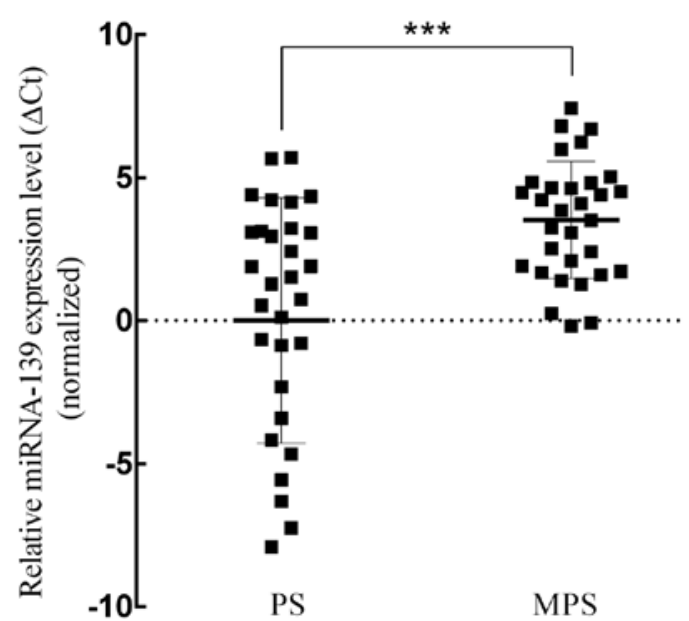

Figure 3. The average $\Delta \mathrm{Ct}$ value of miRNA-139 in the plasma sample of HCC patients was 0.009 , which was significantly lower than that of 3.516 in the matched plasma sample of the chronic HBV-hepatitis patient group. ${ }_{* * * *} \mathrm{p}<0.001$

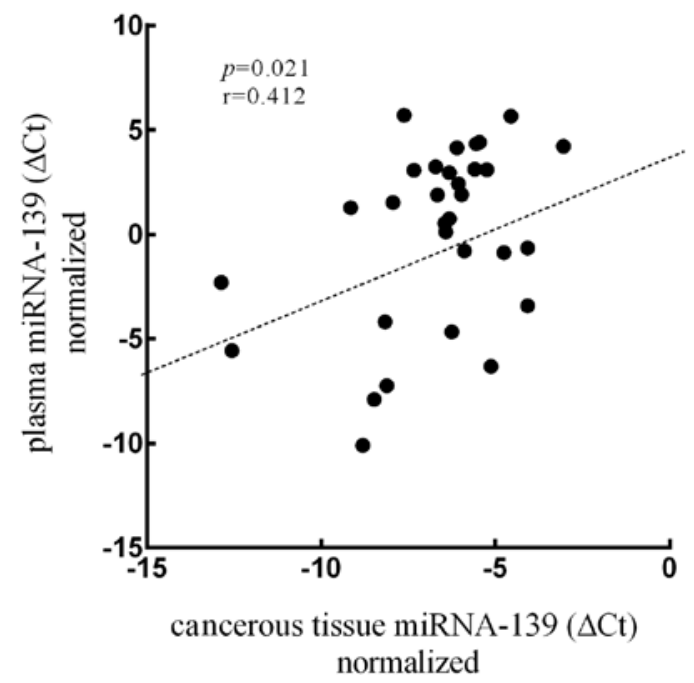

Figure 4. The correlation curve between miRNA-139 expression level in the plasma sample and that in the cancerous tissue of HCC patients. The correlation coefficient was 0.412 , and the p-value was 0.021 .

those in the NT group, with an average $\Delta \mathrm{Ct}$ value of -6.704 vs. -3.490 ( $\mathrm{p}<0.001, \mathrm{t}=-6.785$; Fig. 2). Subsequently, we analyzed miRNA-139 expression level in the PS and MPS groups. The results demonstrated that miRNA-139 expression level in the PS group was significantly lower than that in the MPS group (average $\Delta \mathrm{Ct}$ value: 0.009 vs. $3.516, \mathrm{p}<0.001$, $\mathrm{t}=-4.117$; Fig. 3 ). Meanwhile, the expression quantity of miRNA-139 in plasma was positively correlated to that in cancerous tissues $(\mathrm{p}=0.021$, $\mathrm{r}=0.412$; Fig. 4).

The diagnostic value of miRNA-139, AFP, and combination of miRNA-139 and AFP for HCC. ROC curve was performed. To evaluate the differentiating power of miRNA-139, plasma miRNA-139 expression level was confirmed to be weakly correlated with serum AFP value $(p=0.024, r=0.406)$. Plasma miRNA-139 was able to identify HCC patients from $\mathrm{CH}$ patients with AUC value of 0.761 (95\% CI, 0.643-0.878, $\mathrm{p}<0.001$; Fig. 5A). At the cut-off value of -3.240 for plasma miRNA-139, the optimal sensitivity and specificity were 80.6 

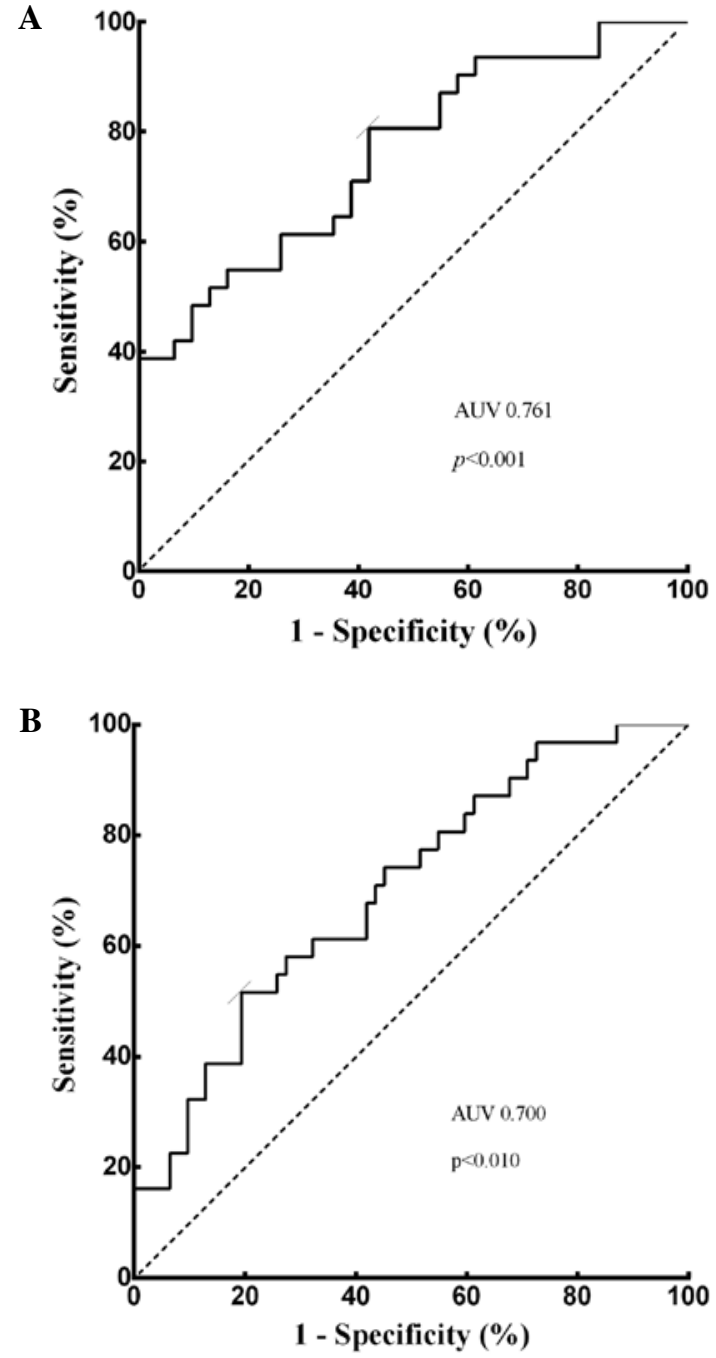

Figure 5. (A) ROC curve of plasma miRNA-139. At the cut-off value of -3.240 for plasma miRNA-139, AUC value was 0.761 and the $95 \%$ confidence interval ranged from 0.643 to 0.878 . The optimal sensitivity and specificity were 80.6 and $58.1 \%$, respectively. (B) ROC curve of serum AFP. At the cutoff value of $17.05 \mathrm{ng} / \mathrm{ml}$ for serum AFP, the AUC value was 0.700 and the $95 \%$ confidence interval ranged from 0.571 to 0.829 . The optimal sensitivity and specificity were 51.6 and $79.3 \%$, respectively. AUC, area under curve ROC, receiver operator characteristic; AFP, $\alpha$-fetoprotein.

and $58.1 \%$, respectively. Subsequently, with the cut-off value of $17.05 \mathrm{ng} / \mathrm{ml}$, the AUC of serum AFP was $0.700(\mathrm{p}<0.010$, 95\% CI, 0.571-0.829; Fig. 5B), while the sensitivity and specificity were 51.6 and $79.3 \%$, respectively, which was consistent with previous studies (13). Similarly, the differentiating power for combination of plasma miRNA-139 with serum AFP was analyzed, and we found that the combination of these two markers improved the power of screening HCC. The combination retrieved a significantly higher sensitivity of 90.3 and specificity of $87.1 \%$, while the AUC increased to 0.770 ( $\mathrm{p}<0.010,95 \%$ CI, 0.654-0.886).

Plasma miRNA-139 correlates with clinicopathological features and 1-year survival analysis of HCC. We examined the correlations between plasma miRNA-139 expression level and some clinical features (Table II). All HCC patients were divided into two groups according to the expression status of miRNA-139: the low expression group $(n=25)$, representing the patients with plasma miRNA-139 level under the optimal

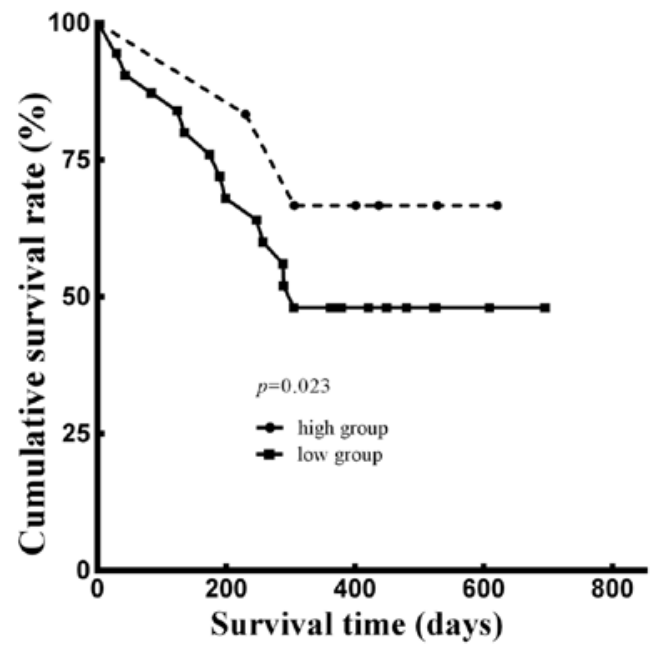

Figure 6. Adjusted for age, gender, weight loss percentage, smoking status, Edmondson-Steiner grading, Child-Pugh grading and CLIP scoring, there is a significant difference in the 1-year survival rates between the high miRNA-139 expression level group and the low group $(\mathrm{p}=0.023)$. The 1-year cumulative survival rate of patients in the high group was $66.7 \%$ with 503 survival days ( \pm 69 days) median survival time, and that in the low expression group was $48.0 \%$, and the median survival time was 439 days ( \pm 50 days).

cut-off of -3.240 , and the high expression group, representing the remaining 6 patients. The results revealed that plasma miRNA-139 expression level was correlated with EdmondsonSteiner grading $(p=0.038)$, serum AFP value $(p=0.043)$, CEA value $(\mathrm{p}=0.034)$ and $\mathrm{DB}$ value $(\mathrm{p}=0.041)$. However, there was no correlation between miRNA-139 expression level and other features, such as age, gender and clinical staging. Furthermore, we analyzed the 1-year survival rate using the Kaplan-Meier method. Adjusted for age, gender, weight loss percentage, smoking status (14), Edmondson-Steiner grading (15), Child-Pugh grading (16) and CLIP scoring (17), there was a significant difference between the two groups $(\mathrm{p}=0.023$; Fig. 6). The Kaplan-Meier analysis indicated that the 1-year cumulative survival rate of patients in the high expression group was $66.7 \%$ with 503 survival days ( \pm 69 days) median survival time, while that in the low expression group was $48.0 \%$, and the median survival time was 439 days ( \pm 50 days).

\section{Discussion}

Comprehensive out-of-hospital surveillance for chronic HBV-hepatitis patients has led to an earlier diagnosis of small lesions being precursors to malignancy (18). However, current existing tumor markers are insufficient to diagnose $\mathrm{HCC}$ at early onset. As a result, HCC is always defined as one of the most common and aggressive malignancies and usually with a poor prognosis worldwide. Although, liver transplantations are considered possible curative therapies, the strict Milan criteria and the limitations of donor availability impede patients from receiving liver transplantations (19). In addition, surgical resection is feasible only if the patient was evaluated with adequate functional liver remnant and solitary mass without major vascular invasion (20). To cure HCC patients at the relatively early stage, serum AFP value has been mostly applied for screening $\mathrm{HCC}$, whereas the sensitivity and specificity are not satisfied. 
Table II. Clinicopathological characteristics of HCC patients categorized according to the plasma miRNA-139 expression level.

\begin{tabular}{|c|c|c|c|}
\hline & \multicolumn{2}{|c|}{ Plasma miRNA-139 expression level } & \multirow[b]{2}{*}{ P-value } \\
\hline & $\begin{array}{c}\text { Low group }(n=25) \\
{[<-3.240(\Delta C t)]}\end{array}$ & $\begin{array}{l}\text { High group }(\mathrm{n}=6) \\
{[\geq-3.240(\Delta \mathrm{Ct})]}\end{array}$ & \\
\hline \multicolumn{4}{|l|}{ Clinical factors } \\
\hline Age (years) & $49 \pm 11$ & $48 \pm 12$ & 0.747 \\
\hline Gender (male/female) & $20 / 5$ & $6 / 0$ & 0.553 \\
\hline WBC (x 109/1) & $5.92 \pm 2.53$ & $6.63 \pm 2.38$ & 0.538 \\
\hline $\mathrm{RBC}\left(\mathrm{x} 10^{12} / \mathrm{l}\right)$ & $4.61 \pm 0.80$ & $4.23 \pm 0.69$ & 0.297 \\
\hline $\operatorname{PLT}\left(\times 10^{9} / 1\right)$ & $135.44 \pm 61.46$ & $101.67 \pm 41.50$ & 0.214 \\
\hline $\mathrm{Hb}(\mathrm{g} / \mathrm{l})$ & $141.40 \pm 20.03$ & $133.50 \pm 23.05$ & 0.405 \\
\hline $\operatorname{ALB}(\mathrm{g} / \mathrm{l})$ & $40.62 \pm 4.22$ & $38.70 \pm 4.92$ & 0.340 \\
\hline $\mathrm{TB}(\mu \mathrm{mol} / \mathrm{l})$ & $22.53 \pm 7.69$ & $19.61 \pm 7.97$ & 0.423 \\
\hline $\mathrm{DB}(\mu \mathrm{mol} / \mathrm{l})$ & $8.78 \pm 5.85$ & $4.97 \pm 3.39$ & $0.041^{\mathrm{a}}$ \\
\hline $\mathrm{IB}(\mu \mathrm{mol} / \mathrm{l})$ & $13.71 \pm 6.92$ & $14.24 \pm 5.60$ & 0.843 \\
\hline $\operatorname{ALT}(\mathrm{U} / \mathrm{l})$ & $44.28 \pm 24.28$ & $50.17 \pm 13.48$ & 0.574 \\
\hline AST (U/l) & $55.04 \pm 31.22$ & $66.33 \pm 27.73$ & 0.424 \\
\hline $\operatorname{ALP}(\mathrm{U} / \mathrm{l})$ & $173.08 \pm 309.04$ & $115.33 \pm 34.00$ & 0.655 \\
\hline GGT (U/l) & $104.84 \pm 96.05$ & $59.33 \pm 28.13$ & 0.265 \\
\hline Operation time (min) & $198 \pm 44$ & $179 \pm 45$ & 0.355 \\
\hline Blood loss (ml) & $728 \pm 570$ & $793 \pm 684$ & 0.810 \\
\hline Liver cirrhosis $(+/-)$ & $16 / 9$ & $4 / 2$ & 0.999 \\
\hline Hypersplenism (+/-) & $13 / 12$ & $3 / 3$ & 0.999 \\
\hline \multicolumn{4}{|l|}{ Tumor-related factors } \\
\hline $\operatorname{AFP}(\mathrm{ng} / \mathrm{ml})$ & $29.50 \pm 934.35$ & $8.80 \pm 13.48$ & $0.043^{a}$ \\
\hline CEA (ng/ml) & $4.18 \pm 2.85$ & $1.55 \pm 0.59$ & $0.034^{\mathrm{a}}$ \\
\hline CA19-9 (U/ml) & $22.21 \pm 16.89$ & $15.82 \pm 10.00$ & 0.384 \\
\hline Child-Pugh grading $(\mathrm{A} / \mathrm{B})$ & $21 / 4$ & $5 / 1$ & 0.999 \\
\hline Tumor no. (single/multiple) & $22 / 3$ & $4 / 2$ & 0.241 \\
\hline Tumor size $(\mathrm{cm})$ & $8.24 \pm 3.2$ & $7.63 \pm 4.22$ & 0.698 \\
\hline Vascular invasion $(+/-)$ & $1 / 24$ & $1 / 5$ & 0.366 \\
\hline Edmondson-Steiner grading (I/II+III) & $2 / 23$ & $3 / 3$ & $\mathbf{0 . 0 3 8}^{\mathrm{a}}$ \\
\hline TNM staging (I/II/III) & $10 / 1 / 14$ & $2 / 2 / 2$ & 0.171 \\
\hline Okuda staging (1/2) & $11 / 14$ & $3 / 3$ & 0.872 \\
\hline BCLC staging $(\mathrm{A} / \mathrm{B} / \mathrm{C})$ & $11 / 7 / 7$ & $3 / 1 / 2$ & 0.958 \\
\hline CLIP scoring $(1 / 2 / 3)$ & $11 / 11 / 3$ & $4 / 2 / 0$ & 0.823 \\
\hline
\end{tabular}

All HCC patients were divided into two groups according to the expression status of miRNA-139: the low expression group ( $\mathrm{n}=25$ ), representing the patients with plasma miRNA-139 level under the aforementioned optimal cut-off of -3.240 , and the high expression group. Plasma miRNA-139 expression level is correlated with Edmondson-Steiner grading $(\mathrm{p}=0.038)$, serum AFP value $(\mathrm{p}=0.043), \mathrm{CEA}$ value $(\mathrm{p}=0.034)$, and DB value $(\mathrm{p}=0.041)$. However, there is no correlation between miRNA-139 expression level and other features, such as age, gender and clinical staging. " $\mathrm{p}<0.050$. HCC, hepatocellular carcinoma; WBC, white blood cell; RBC, red blood count; PLT, platelets; Hb, hemoglobin; ALB, albumin; TB, total bilirubin; DB, direct bilirubin; IB, indirect bilirubin; ALT, alanine transaminase; AST, aspartate aminotransferase; ALP, alkaline phosphatase; GGT, $\gamma$-glutamyl transpeptidase; AFP, $\alpha$-fetoprotein; CEA, carcinoembryonic antigen; CA19-9, carbohydrate antigen 19-9; BCLC staging, Barcelona Clinic Liver Cancer staging; CLIP scoring, the Cancer of the Liver Italian Program scoring.

Previous results revealed that miRNAs in blood circulation were markedly stable (21) and they may be potential diagnostic and prognostic factors in diverse diseases, particularly in the field of malignant neoplasms. Since the serum miRNA-21 was identified as the first one for diagnosing patients with diffuse large B cell lymphoma, and was associated with recurrencefree survival (22), circulating miRNAs were frequently studied as potential biomarkers for several types of cancer. To date, some miRNAs have been reported to be associated with the diagnosis and prognosis of liver cancer (23).

In the present study, we found that miRNA-139 expression was significantly lower in hepatocellular cancerous tissues than in their peritumoral non-cancerous tissues in miRNA microarray analysis. Then, the miRNA-139 expression profile 
was further detected in the tissue and plasma samples of $31 \mathrm{HCC}$ patients and another 31 age- and gender-matched $\mathrm{CH}$ patients. Our results demonstrated that the average value of miRNA-139 expression level in HCC patients was 0.009, which was significantly lower than the value of 3.516 in $\mathrm{CH}$ patients. To our knowledge, the present study is the first one to identify miRNA-139 expression profiles both in HCC patients and $\mathrm{CH}$ patients. Low miRNA-139 level has been reported in digestive malignant tumor, such as gastric (24) and colorectal cancer (25), and also in adrenocortical carcinomas (26), parathyroid cancer (27), and squamous cell carcinoma in tongue (28). Thus, we consider that the downregulation of plasma miRNA-139 expression may be a common event in malignancies. In terms of liver cancer, Wong et al (29) first reported low-expression of miRNA-139 in HCC may suppress metastasis and progression of cancer cells by downregulating Rho-kinase 2. The following year, Professor Wong's study team indicated that miRNA-139 is a tumor-suppressor miRNA, and enhancer of zeste homolog 2 (EZH2) may be responsible for the downregulation of the miRNAs in human HCCs (30). Based on the aforementioned findings, we further hypothesized that miRNA-139 may be excreted into the extracellular space and it may also be observed in blood. In the present study, we confirmed the correlation between miRNA-139 expression in plasma and cancerous tissue. Many studies have revealed the correlation between plasma miRNAs and cancerous tissue miRNAs, and have proposed the viewpoint that tissue intracellular miRNAs would be released into circulation during tumorigenesis, which was accompanied by pathological injury or cellular destruction $(21,22)$. However, due to the sample size and the inconsistent data of certain patients in correlation analysis, the correlation coefficient of miRNA-139 expression in plasma and cancerous tissue was only 0.412 . Further verification of our findings is required in a large population.

ROC curves for the diagnostic value of plasma miRNA-139 yielded an AUC of 0.761 with the sensitivity of $80.6 \%$ and the specificity of $58.1 \%$ in HCC diagnosis. Meanwhile, in the same population, AUC of serum AFP was 0.700 at the cut-off value of $17.05 \mathrm{ng} / \mathrm{ml}$, with the sensitivity of 51.6 and the specificity of $79.3 \%$. However, the combination of miRNA-139 and AFP increased the sensitivity to 90.3 and the specificity to $87.1 \%$, while the AUC was 0.770 , which was considerably better than miRNA-139 or AFP alone.

For a better understanding of the clinical implications of plasma miRNA-139, we also examined the correlations between plasma miRNA-139 expression level and clinical features. Edmondson-Steiner grading, which classifies HCC according to HCC cell differentiation, morphology, and mitotic phase (15), was negatively associated with plasma miRNA-139 expression. The plasma miRNA-139 expression level decreased with the increase of Edmondson-Steiner grading. Edmondson-Steiner grade was always considered to be positively correlated with the invasion and tumor recurrence may account for the dismal prognosis of patients with poorly differentiated HCC (31), indicating that miRNA-139 acted as the protective agent for $\mathrm{HCC}$ from differentiation. However, no association was found between miRNA-139 and clinical staging or scoring system (including TNM staging, Okuda scoring, BCLC staging, and CLIP scoring). Furthermore, the 1-year survival analysis showed the HCC patients with lower
miRNA-139 expression presented shorter survival time, indicating miRNA-139 may be a potential indicator of survival prediction for $\mathrm{HCC}$ patients.

In conclusion, miRNA-139 is downregulated in both cancerous tissue and plasma of HCC. The plasma miRNA-139 is a possible diagnostic biomarker for identifying $\mathrm{HCC}$ patients while combined with other biomarkers, it is also a prognostic factor for indicating patient survival. However, the mechanisms of miRNA-139 dysregulation due to primary expression or secondary changes, require further investigation.

\section{Acknowledgements}

This study was supported by grant nos. 81172287 and 30901457 from the National Natural Science Foundation of China.

\section{References}

1. Jemal A, Bray F, Center MM, Ferlay J, Ward E and Forman D: Global cancer statistics. CA Cancer J Clin 61: 69-90, 2011.

2. Srivatanakul P, Sriplung $H$ and Deerasamee S: Epidemiology of liver cancer: an overview. Asian Pac J Cancer Prev 5: 118-125, 2004.

3. Thorgeirsson SS and Grisham JW: Molecular pathogenesis of human hepatocellular carcinoma. Nat Genet 31: 339-346, 2002.

4. Marrero JA and Lok AS: Newer markers for hepatocellular carcinoma. Gastroenterology 127 (Suppl 1): S113-S119, 2004

5. Jannot G and Simard MJ: Tumour-related microRNAs functions in Caenorhabditis elegans. Oncogene 25: 6197-6201, 2006.

6. Lujambio A and Lowe SW: The microcosmos of cancer. Nature 482: 347-355, 2012.

7. Meng F, Henson R, Lang M, et al: Involvement of human micro-RNA in growth and response to chemotherapy in human cholangiocarcinoma cell lines. Gastroenterology 130: 2113-2129, 2006.

8. Xia L, Zhang D, Du R, et al: miR-15b and miR-16 modulate multidrug resistance by targeting BCL2 in human gastric cancer cells. Int J Cancer 123: 372-379, 2008.

9. Budhu A, Jia HL, Forgues M, et al: Identification of metastasisrelated microRNAs in hepatocellular carcinoma. Hepatology 47: 897-907, 2008.

10. Calin GA, Ferracin M, Cimmino A, et al: A MicroRNA signature associated with prognosis and progression in chronic lymphocytic leukemia. N Engl J Med 353: 1793-1801, 2005.

11. Luo X, Burwinkel B, Tao S and Brenner H: MicroRNA signatures: novel biomarker for colorectal cancer? Cancer Epidemiol Biomarkers Prev 20: 1272-1286, 2011.

12. Schmittgen TD and Livak KJ: Analyzing real-time PCR data by the comparative $\mathrm{C}_{\mathrm{T}}$ method. Nat Protoc 3: 1101-1108, 2008.

13. Marrero JA, Feng Z, Wang Y, et al: $\alpha$-fetoprotein, des- $\gamma$ carboxyprothrombin, and lectin-bound $\alpha$-fetoprotein in early hepatocellular carcinoma. Gastroenterology 137: 110-118, 2009.

14. Shih WL, Chang HC, Liaw YF, et al: Influences of tobacco and alcohol use on hepatocellular carcinoma survival. Int $\mathrm{J}$ Cancer 131: 2612-2621, 2012.

15. Edmondson HA and Steiner PE: Primary carcinoma of the liver: a study of 100 cases among 48,900 necropsies. Cancer 7: 462-503, 1954.

16. Child CG and Turcotte JG: Surgery and portal hypertension. Major Probl Clin Surg 1: 1-85, 1964.

17. No authors listed: A new prognostic system for hepatocellular carcinoma: a retrospective study of 435 patients: the Cancer of the Liver Italian Program (CLIP) investigators. Hepatology 28: 751-755, 1998.

18. Sherman M: Approaches to the diagnosis of hepatocellular carcinoma. Curr Gastroenterol Rep 7: 11-18, 2005.

19. Forner A, Llovet JM and Bruix J: Hepatocellular carcinoma. Lancet 379: 1245-1255, 2012.

20. Maluccio $M$ and Covey $A$ : Recent progress in understanding, diagnosing, and treating hepatocellular carcinoma. CA Cancer J Clin 62: 394-399, 2012.

21. Mitchell PS, Parkin RK, Kroh EM, et al: Circulating microRNAs as stable blood-based markers for cancer detection. Proc Natl Acad Sci USA 105: 10513-10518, 2008. 
22. Lawrie CH, Gal S, Dunlop HM, et al: Detection of elevated levels of tumour-associated microRNAs in serum of patients with diffuse large B-cell lymphoma. Br J Haematol 141: 672-675, 2008.

23. Tomimaru Y, Eguchi $\mathrm{H}$, Nagano $\mathrm{H}$, et al: Circulating microRNA-21 as a novel biomarker for hepatocellular carcinoma. J Hepatol 56: 167-175, 2012.

24. Bao W, Fu HJ, Xie QS, et al: HER 2 interacts with CD44 to up-regulate CXCR4 via epigenetic silencing of microRNA-139 in gastric cancer cells. Gastroenterology 141: 2076-2087, 2011.

25. Shen K, Liang Q, Xu K, et al: MiR-139 inhibits invasion and metastasis of colorectal cancer by targeting the type I insulin-like growth factor receptor. Biochem Pharmacol 84 320-330, 2012.

26. Schmitz KJ, Helwig J, Bertram S, et al: Differential expression of microRNA-675, microRNA-139-3p and microRNA-335 in benign and malignant adrenocortical tumours. J Clin Pathol 64: 529-535, 2011
27. Corbetta S, Vaira V, Guarnieri V, et al: Differential expression of microRNAs in human parathyroid carcinomas compared with normal parathyroid tissue. Endocr Relat Cancer 17: 135-146, 2010.

28. Wong TS, Liu XB, Wong BY, Ng RW, Yuen AP and Wei WI: Mature miR-184 as potential oncogenic microRNA of squamous cell carcinoma of tongue. Clin Cancer Res 14: 2588-2592, 2008.

29. Wong CC, Wong CM, Tung EK, et al: The microRNA miR-139 suppresses metastasis and progression of hepatocellular carcinoma by down-regulating Rho-kinase 2. Gastroenterology 140: 322-331, 2011.

30. Au SL, Wong CC, Lee JM, et al: Enhancer of zeste homolog 2 epigenetically silences multiple tumor suppressor microRNAs to promote liver cancer metastasis. Hepatology 56: 622-631, 2012.

31. KerCG,ChenHY,Chen KS, et al: Clinical significance of cell differentiation in hepatocellular carcinoma. Hepatogastroenterology 50 : 475-479, 2003 